\title{
Indagine conoscitiva sui fabbisogni formativi del personale tecnico-sanitario
}

\author{
Marina Crovatto', Graziella Gava', CoTeLab: Enrico Magliano, Anna Camaggi, \\ Daniela Marchetti, Graziella Marin, Giuseppe Miragliotta, Irene Panecaldo, Lidia Ricci, \\ Stefania Pirazzoli, Ferdinando Salemi, Riccardo Smeraglia, Alberto Spanò, Erminio Torresani
}

'Struttura Operativa Semplice di Immunologia Clinica e Virologia, Dipartimento di Medicina di Laboratorio Azienda Ospedaliera Santa Maria degli Angeli, Pordenone

Key words: technicians, continuing education. quality control, education program, molecular biology, epidemiology, biological hazard, hospital infection.

Indagine conoscitiva sui fabbisogni formativi del personale tecnico-sanitario

Survey on the technician's needs for continuing medical education

\section{SUMMARY}

The primary objective of AMCLI is to provide efficient, high quality continuing education. The CoTeLab has prepared a questionnaire including the main groups of interest of Laboratory Technologists, either of a specific or general nature, as well as other eventual arguments, suggestions and observations in order to understand the real necessity of training. The questionnaire was answered by 464 technicians of which 7 I \% females and $29 \%$ males, most of interviewed were between $4 \mathrm{I}$ and 45 years of age with variable experience. Scolarity was not homogeneous due to Italian evolution in the past years.

The necessity to up-date knowledge in the laboratory in which day-to-day work is performed and an elevated interest in molecular biology was evidenced.

Quality control, biological hazard and hospital infections were the arguments most requested.

The procedure of accreditation and/or certification already obtained in many laboratories, and the problems involved in new technologies are certainly factors that bring attention to Quality Control.

The response in respect of biological hazards is due to the implementation of DL 626/94 and its successive modifications.

Hospital infections have always been an important subject of microbiology and have gained much attention thanks to the new technologies offered (molecular epidemiology)

Theoretical and practical training is mainly requested and evidences the technician's need to understand routine procedures and not only to perform them.

New technologies are the general arguments most requested and preferred.

Surprisingly long distance education is not requested, probably since it has not yet been activated and the modality is not clear.

In conclusion this study has hallowed us to have a reliable perspective of the educational needs of technologists and will permit us to gradually construct a high quality, efficient continuing education program that will certainly give positive results in the total quality of results referred to physicians.

\section{INTRODUZIONE}

In questi ultimi anni c'è stato uno sviluppo tecnologico esplosivo in tutti i campi della medicina ed un accumularsi rapido e continuo di nuove conoscenze che hanno determinato cambiamenti radicali nel modo di affrontare e risolvere i problemi ed hanno aperto prospettive diverse e certamente impensabili fino a qualche anno fa. Uno dei campi in cui l'influenza della spinta innovativa si è fatta maggiormente sentire è quello della Microbiologia, dove l'introduzione di tecniche quali ad esempio quelle molecolari ha rivoluzionato l'approccio alla diagnosi di laboratorio delle malattie infettive $(5,9,10,14)$. Parallelamente all'evoluzione tecnologica la globalizzazione ha favorito i flussi migratori e la rapida mobilità delle popolazioni, con conseguenti modificazioni dei comportamenti umani. Altri fattori quali le modificazioni dell'ambiente, l'introduzione di nuove terapie (es. terapie anticancro) e dei trapianti hanno contribuito a modificare gli equilibri favorendo l'insorgenza di nuove malattie infettive e/o inducendo modificazioni di processi infettivi preesistenti. Tutto questo, associato alla progressiva diminuzione della disponibilità di risorse umane e materiali ha imposto una pesante riorganizzazione in tutto l'ambito sanitario (non solo a livello italiano, ma mondiale), soprattutto nei servizi diagnostici ed ha accentuato l'esigenza di rivedere i processi formativi delle diverse figure professionali e di istituire dei percorsi di educazione continua atti a mantenere gli standard pro- 
fessionali, a far fronte alle innovazioni ed a tutte le problematiche che la riorganizzazione comporta $(1-4,6-8,11-13,15,19,20)$.

L'attivazione del programma nazionale per l'Educazione Continua in Medicina (ECM) ha proprio lo scopo di fornire a tutte le figure professionali eventi formativi qualitativamente ottimali e un'ampia gamma di possibilità di scelta per guadagnare i crediti necessari a testimoniare il miglioramento ed il mantenimento degli standard professionali (17).

Tra i vari provider di eventi formativi le Associazioni Scientifiche hanno un peso indiscutibile e l'obbiettivo principale che si devono prefissare è sì quello della qualità ma anche quello dell'efficacia ossia dell'indirizzare gli eventi sulla base dei fabbisogni formativi specifici degli operatori $(16,17)$.

L'AMCLI ha sempre perseguito quale obbiettivo fondamentale quello di dare ai suoi associati una educazione continua mirata, efficace e qualitati- vamente valida con l'ausilio di Comitati e di Gruppi di lavoro: l'attività fino ad ora svolta ne è una chiara testimonianza. Per poter intervenire in modo più incisivo e mirato e raggiungere tutte le categorie professionali che operano nel campo della Microbiologia, ha istituito nell'aprile del 2002 il CoTeLab (Comitato di Studio per lo Sviluppo Professionale Continuo dei Tecnici di Laboratorio) che ha il compito di curare l'attività formativa dei tecnici nell'ottica dell'ECM e favorire la loro crescita culturale e pratica specifica. Il primo impegno che il Comitato si è assunto è stato quello di approfondire quali sono i reali fabbisogni formativi dei tecnici per poter predisporre un progetto formativo adeguato, efficace e di qualità, da attuare nell'immediato futuro.

\section{METODI}

È stato preparato un questionario nel quale si è cercato di inquadrare i principali campi di interesse per il tecnico di laboratorio microbiologico (figura I) sia a carattere specifico che generale

\section{INDAGINE CONOSCITIVA}

Fabbisogni formativi del personale tecnico-sanitario

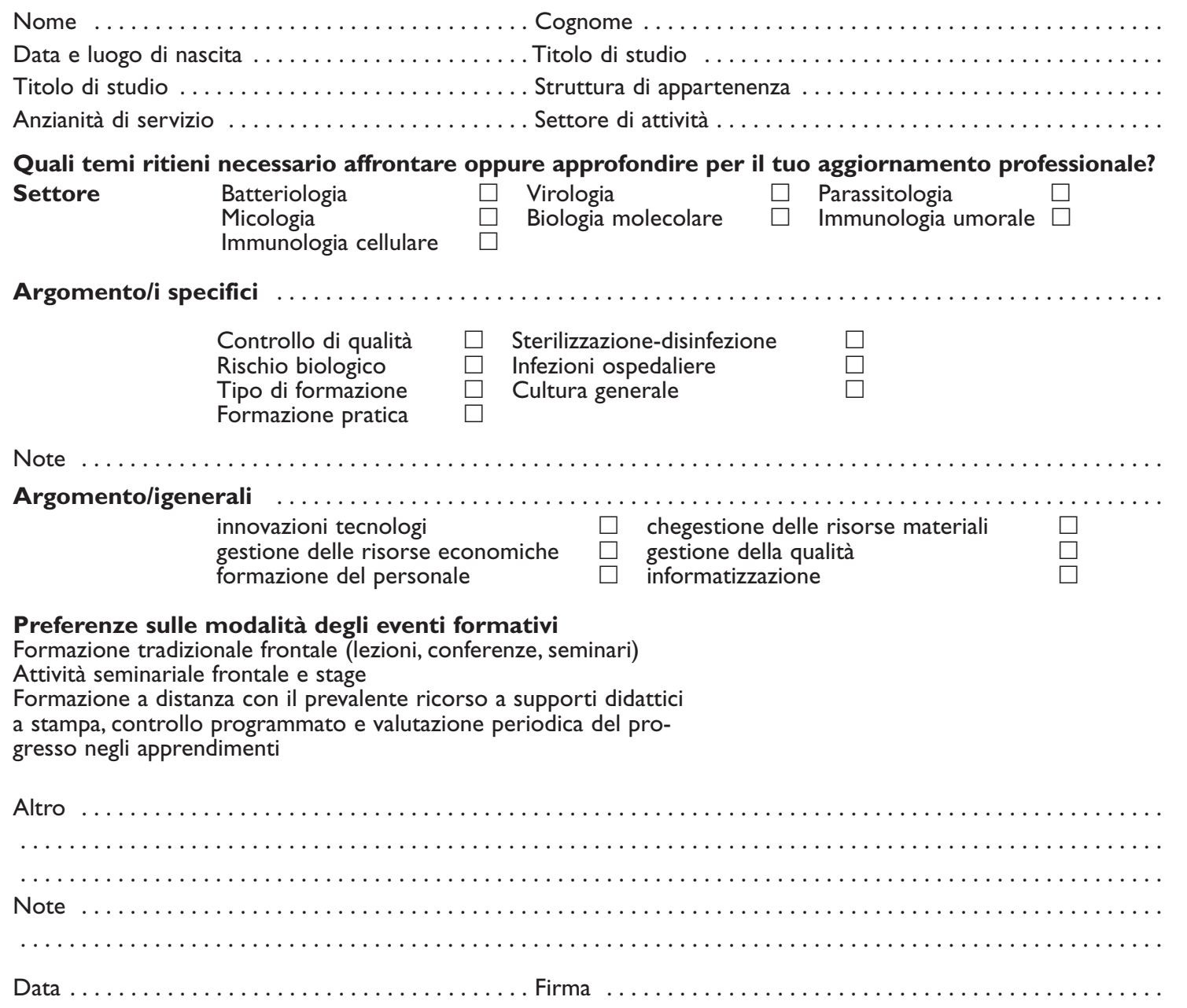

Figura I. 
dando anche spazio ad eventuali argomenti aggiuntivi, a suggerimenti ed osservazioni in modo da arricchire il bagaglio di informazioni previsto e coprire il più possibile il range di fabbisogni.

La parte finale del questionario è stata riservata alle preferenze sulle modalità con cui devono essere attuati gli eventi formativi in modo da individuare quelle più congeniali ed agevoli.

Modalità di diffusione: il questionario è stato inserito nel sito AMCLI da luglio 2002 a gennaio 2003 e su "Notizie AMCLI" di agosto 2002.

Elaborazione dati: i dati riportati sulle schede pervenute entro il 31 gennaio 2003 sono stati inseriti in computer ed elaborati utilizzando il programma excel. Sono stati quindi discussi e commentati con tutti i componenti del CoTeLab.

\section{RISULTATI}

Caratteristiche partecipanti

Hanno aderito all'iniziativa 464 tecnici dei quali il $71 \%$ di sesso femminile e il $29 \%$ di sesso maschile (Grafico 1). La prevalenza del sesso femminile è omogeneamente presente su tutto il territorio nazionale.

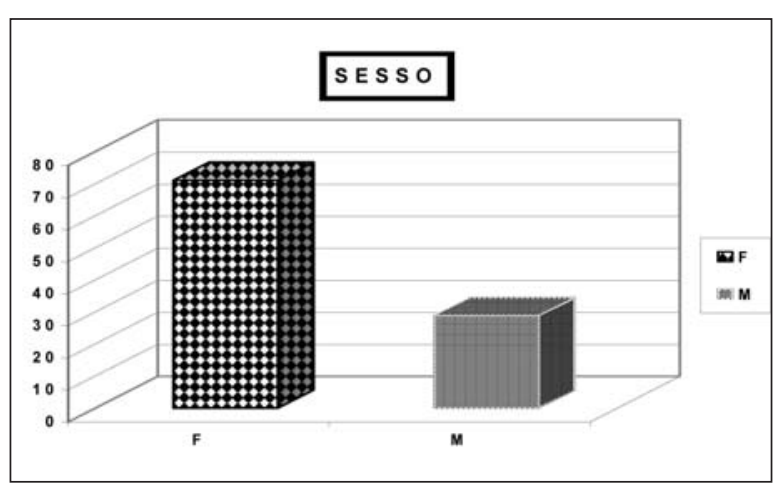

\section{Grafico I}

I 464 questionari sono pervenuti dalle varie regioni nelle seguenti percentuali:

\begin{tabular}{lclc}
\hline Basilicata & $0.2 \%$ & Marche & $0.4 \%$ \\
\hline Calabria & $4.3 \%$ & Piemonte & $4.1 \%$ \\
\hline Campania & $22.6 \%$ & Puglia & $1.1 \%$ \\
\hline Emilia Romagna & $26.7 \%$ & Toscana & $0.2 \%$ \\
\hline Friuli-Venezia Giulia & $14.7 \%$ & Trentino-Alto Adige & $10.6 \%$ \\
\hline Lazio & $5.0 \%$ & Umbria & $0.4 \%$ \\
\hline Lombardia & $4.1 \%$ & Veneto & $5.2 \%$ \\
\hline Molise & $0.2 \%$ & & \\
\hline
\end{tabular}

La distribuzione dell'età anagrafica ha un andamento praticamente di tipo "gaussiano" (Grafico 2), con una numerosità più elevata tra i 41 e 45 anni e tendente ad un rapido calo per le fasce di età superiore. Il picco può essere spiegabile con il fatto che con il boom della crescita della medicina di laboratorio e della microbiologia negli ultimi 30 anni c'è stato un aumento graduale di richiesta di personale tecnico per cui gli "over 45" sono di meno rispetto ai più giovani. Va anche tenuto presente che fino a qualche anno fa era possibile il pensionamento con anzianità di servizio piuttosto bassa, per cui anche fra $\mathrm{i}$ tecnici in molti casi c'è stata la scelta della risoluzione del rapporto di lavoro.

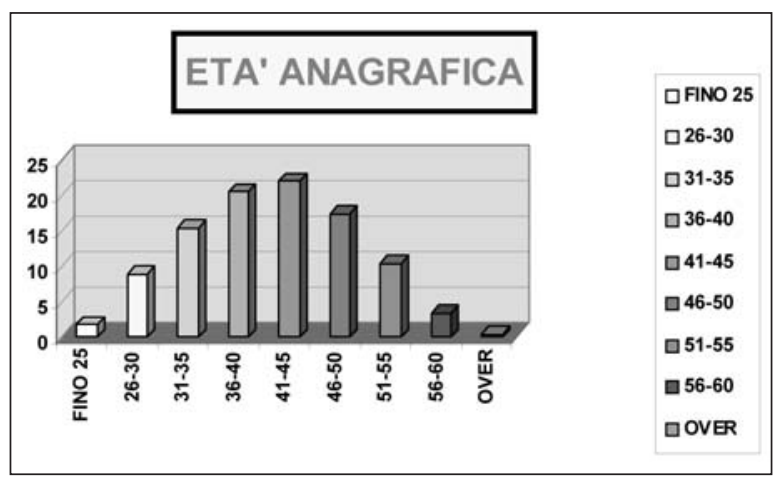

Grafico 2

L'anzianità di servizio non segue lo stesso andamento dell'età anagrafica (Grafico 3) per vari motivi: in molti casi si è osservato che prima di arrivare alla professione di tecnico vi sono state esperienze lavorative diverse. Un'ulteriore spiegazione a carattere più generale sta anche nel fatto che vi sono stati periodi di grandi assunzioni seguiti da periodi di saturazione di tutti i posti disponibili.

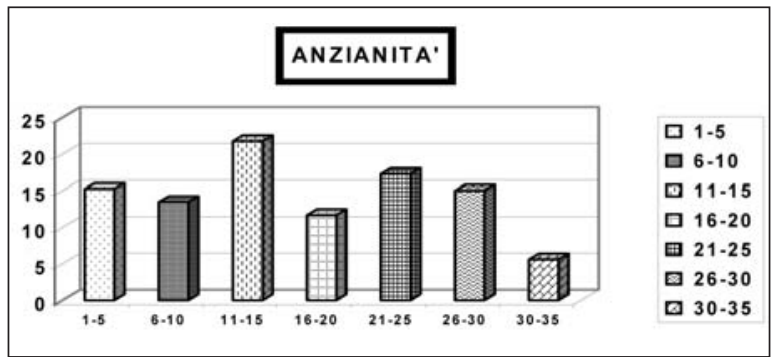

Grafico 3

Il panorama relativo alla scolarità (Grafico 4) è forse il meno omogeneo di tutte le altre figure professionali operanti in sanità, in quanto ha subito molti cambiamenti nel corso degli ultimi anni. $\mathrm{Si}$ è passati dalla licenza di scuola media inferiore seguita da un breve corso formativo richiesto fino a 25 anni fa, ai 5 anni di scuola professionale riconosciuti fino a circa 15 anni fa, ai vari diplomi di scuola media superiore seguiti da corsi formativi ospedalieri o universitari di uno,due o tre anni, per arrivare ai diplomi universitari e poi alla laurea triennale che è stata istituita, per l'e- 
sercizio della professione, nel 2000. Proprio a causa di questa progressiva evoluzione, attualmente si trovano a convivere professionisti che hanno avuto iter formativi diversi.

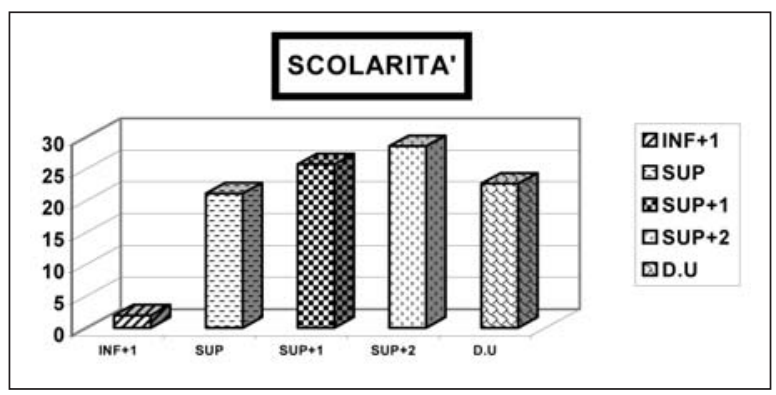

Grafico 4.

Interpretazione legenda del grafico:

$\mathrm{INF}+1$ : scuola media inferiore + corso formativo di qualche mese, al massimo un anno

SUP: scuola professionale di 5 anni ad indirizzo specifico

SUP +1 : diploma di scuola media superiore +1 anno di corso

SUP+2: diploma di scuola media superiore +2 anni di corso

D.U.: diploma di scuola media superiore +3 anni di corso di diploma universitario oggi sostituito da laurea triennale

Poiché in alcuni casi mancava la definizione esatta della scolarità, 1'iter formativo è stato ricostruito in base all'età ed all'anzianità di servizio.

La distribuzione in rapporto alla tipologia del laboratorio di appartenenza (Grafico 5) evidenzia che il $43 \%$ dei tecnici che hanno risposto lavora in un laboratorio di Microbiologia, il 38\% in un laboratorio multidisciplinare in cui comunque è contemplata la microbiologia, il $18 \%$ in laboratori dove non c'è la microbiologia (nel grafico corrispondono ad ALTRO). L'1\% opera in laboratorio Virologia.

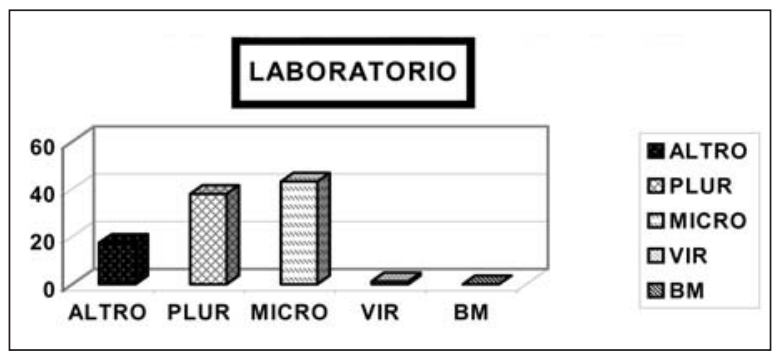

Grafico 5.

Il 35\% lavora in settori multidisciplinari occupandosi pertanto anche di biochimica clinica, oltre che di microbiologia, il 21\% lavora in batteriolo- gia, il 14\% si occupa di discipline diverse dalla microbiologia, il 13\% opera in immunologia e 1 ' $11 \%$ in virologia. Solo il 4\% lavora nel settore di biologia molecolare. Il $2 \%$ non ha segnalato il settore di lavoro. (Grafico 6).

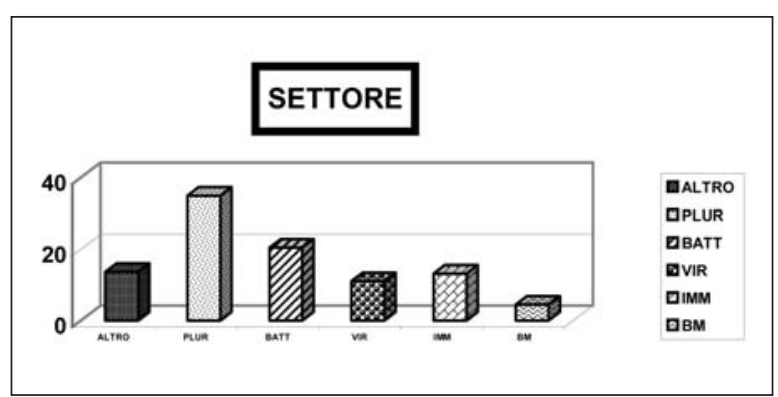

Grafico 6.

\section{Risposte}

Nel Grafico 7 sono evidenziate le risposte relative al settore in cui si richiede l'approfondimento: il $60 \%$ ha espresso preferenza per la Batteriologia, il 59\% per la Biologia molecolare, il $31 \%$ per l'Immunologia cellulare, il $27 \%$ per l'Immunologia umorale e per la Micologia, il $22 \%$ per la Parassitologia e il $46 \%$ per la Virologia.

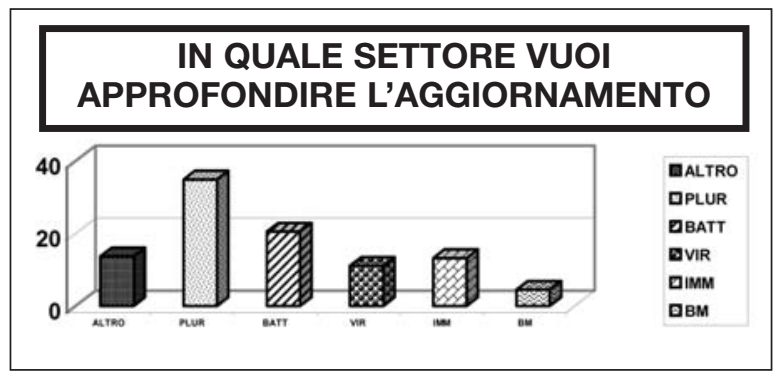

\section{Grafico 7.}

Analizzando le risposte in relazione al posto di lavoro è emerso che la maggior parte dei partecipanti vuole approfondire le conoscenze relative al settore in cui opera, ad esempio chi lavora in batteriologia vuole approfondire le conoscenze in questo settore. C'è invece un gradimento trasversale elevato $(59 \%)$ per la biologia molecolare, sicuramente per l'importanza che sta assumendo sempre di più nell'ambito della diagnostica e per la possibilità praticamente illimitata di applicazione ma anche per le peculiarità che la contraddistinguono sia dal punto di vista concettuale che applicativo.

Nell'ambito dei vari settori (Grafico 8) il 49\% dei tecnici ha espresso preferenza per il Controllo di qualità, il 18\% per la sterilizzazione-disinfezione, il $48 \%$ per il rischio biologico, il $41 \%$ per le infe- 
zioni ospedaliere, il $16 \%$ ha segnalato altro. In "altro" sono inclusi gli argomenti riportati in Tabella 1.

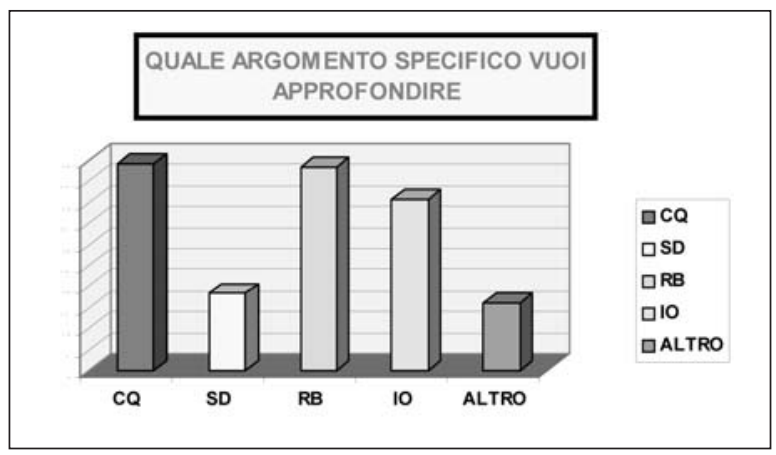

Grafico 8.

Prevale l'esigenza di aggiornamento sulle tecniche più nuove della biologia molecolare (realtime, microarrays, sequenziamento) applicate al settore in cui il tecnico opera (N. 20). Un altro tema su cui viene puntata maggiormente l'attenzione è quello relativo alle infezioni da Micobatteri (N. 10), microorganismo tornato prepotentemente alla ribalta soprattutto in soggetti immunodepressi (con infezione da HIV ad es.) e con caratteristiche di multiresistenza al trattamento farmacologico.

Il tipo di formazione richiesta è prevalentemente teorico-pratica (49\%) (Grafico 9) rispetto a quella solo pratica $(26 \%)$ o solo teorica $(17 \%)$. Analizzando le preferenze in rapporto all'età emerge che una formazione di tipo prettamente pratico è preferita dai più giovani. Questo è un dato che risponde alle attese, dato che i più gio- vani hanno sicuramente una preparazione teorica maggiore rispetto ai più anziani grazie all'iter formativo a cui sono stati sottoposti.

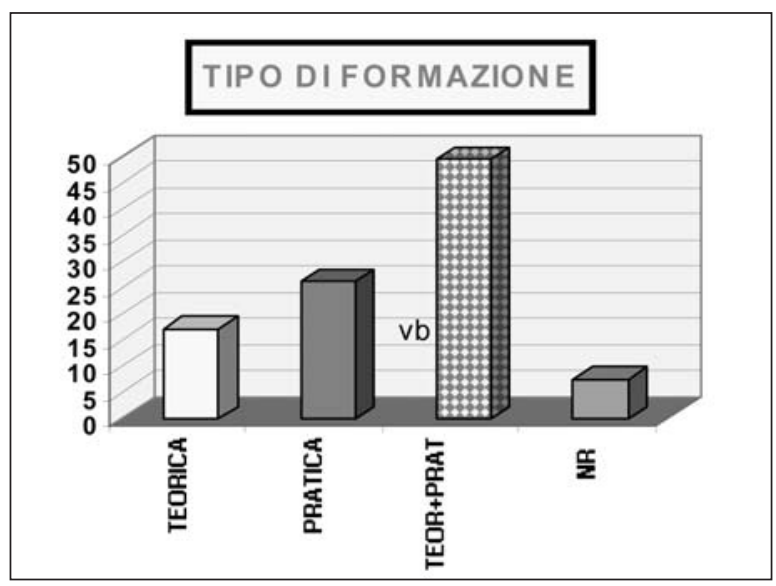

Grafico 9.

È interessante notare (Grafico 10) come il 71\% dei tecnici senta la necessità di aggiornamento sulle innovazioni tecnologiche ed il 49\% esprima l'esigenza di approfondire le conoscenze sull'uso dei programmi informatici più diffusi e dei programmi gestionali di uso quotidiano (PC). L'interesse per la qualità in senso globale e per la partecipazione attiva all'ECM è equivalente: $37 \%$ e $38 \%$ rispettivamente. Solo nel $21 \%$ dei casi viene richiesto l'approfondimento della gestione del movimento di magazzino e di scadenze che è di competenza del personale tecnico. Probabilmente i sistemi gestionali computerizzati dei magazzini che ormai in ogni laboratorio sono stati introdotti, sono a regime ed i training a cui il

Tabella I

\begin{tabular}{|c|c|c|c|}
\hline \multicolumn{4}{|l|}{ ARGOMENTI SPECIFICI } \\
\hline Anaerobi & 5 & Malattia di Lyme & 2 \\
\hline Autoimmunità & 6 & Malattie esantematiche & 2 \\
\hline $\begin{array}{l}\text { Biologia molecolare (PCR quali e quantitativa,real time, } \\
\text { microarrays, sequenziamento, genetica molecolare) } \\
\text { applicata a settori specifici }\end{array}$ & 20 & Malattie ereditarie & I \\
\hline Citofluorimetria in oncoematologia & I & Markers tumorali & I \\
\hline Colture cellulari & 2 & Meccanismi di resistenza agli antibiotici & 3 \\
\hline Controllo di qualità per apparecchiature e plastiche & I & Micobatteri & 10 \\
\hline Epatiti & 4 & Microbiologia degli alimenti & 3 \\
\hline Esami urgenti in reperibilità & I & Microbiologia delle acque (indicatori fecali, parassiti) & 7 \\
\hline Flora microbica normale & I & Microscopia & 2 \\
\hline Helicobacter pylori & I & Modalità di prelievo e verifica idoneità dei campioni & I \\
\hline Infezioni della cute & I & Nuove tecniche di laboratorio in Microbiologia & I \\
\hline Infezioni di ferite chirurgiche & $\mathrm{I}$ & Parassitologia tropicale & 2 \\
\hline Infezioni dell'apparato respiratorio & 2 & Patogeni emergenti & 4 \\
\hline Infezioni endoprotesi e cateteri & I & Prevenzione delle infezioni ospedaliere & I \\
\hline Infezioni materno fetali & $\mathrm{I}$ & Problemi più comuni nei vari settori & $\mathrm{I}$ \\
\hline Infezioni nei pazienti in rianimazione & I & Sicurezza nella manipolazione dei materiali biologici & I \\
\hline Infezioni protozoarie & I & Smaltimento rifiuti & 3 \\
\hline Infezioni sessualmente trasmesse & 3 & Tecniche per la diagnosi delle zoonosi & 2 \\
\hline Isolamento e tipizzazione miceti filamentosi & 3 & Tecniche di laboratorio applicate all'uomo e in veterinaria & $\mathrm{I}$ \\
\hline Legionella & 3 & Torch & 3 \\
\hline Liquor & 2 & Valutazione dei risultati & I \\
\hline
\end{tabular}


personale è stato sottoposto sta dando i suoi frutti.

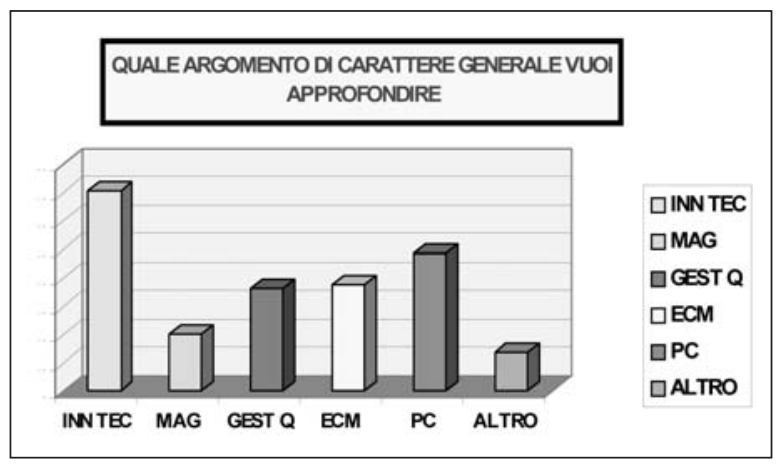

Grafico 10.

Nella voce "Altro" sono inclusi gli argomenti riportati in tabella 2. È evidente il desiderio sia di rivedere alcune conoscenze scolastiche (biologia, chimica, farmacologia, fisica ) che di affrontare nuove acquisizioni soprattutto per chi ha percorso l'iter formativo diversi anni fa (vedi età media dei tecnici) e non ha avuto modo di affrontare certi argomenti (ad es. inglese e statistica)

\section{Tabella 2.}

\begin{tabular}{l|l}
\hline ARGOMENTI GENERALI & \\
\hline Accreditamento, Certificazione & 4 \\
\hline Chimica,biologia,farmacologia & 19 \\
\hline Competenze TSLB & 2 \\
\hline Fisica & 11 \\
\hline Informatica gestionale & 4 \\
\hline Inglese & 17 \\
\hline Matematica & 3 \\
\hline Statistica & 15 \\
\hline Storia della legislazione delle strutture sanitarie & $\mathrm{I}$ \\
\hline
\end{tabular}

Le preferenze maggiori sulle modalità con cui devono essere fatti gli eventi formativi (Grafico 11) vanno alla formazione tradizionale frontale (in Grafico = conferenze) (59\%), seguita dall'attività seminariale frontale e stage (in Grafico $=$ seminari) $(53 \%)$ e dalla formazione "a distanza" $(27 \%)$. La formazione "a distanza" (in Grafico $=$

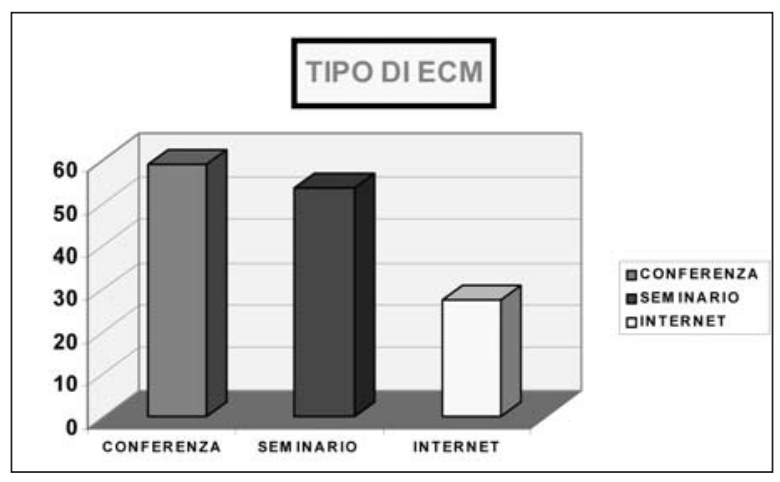

Grafico II. internet) ha trovato scarsa richiesta, probabilmente perché non è ancora impostata ed avviata e quindi non può ancora essere valutata e capita in base alle sue reali potenzialità.

Grafico 11.

Si è voluto anche valutare qual è l'età media di coloro che gradirebbero l'utilizzo di internet come mezzo formativo ed i risultati sono visibili in Grafico 12.

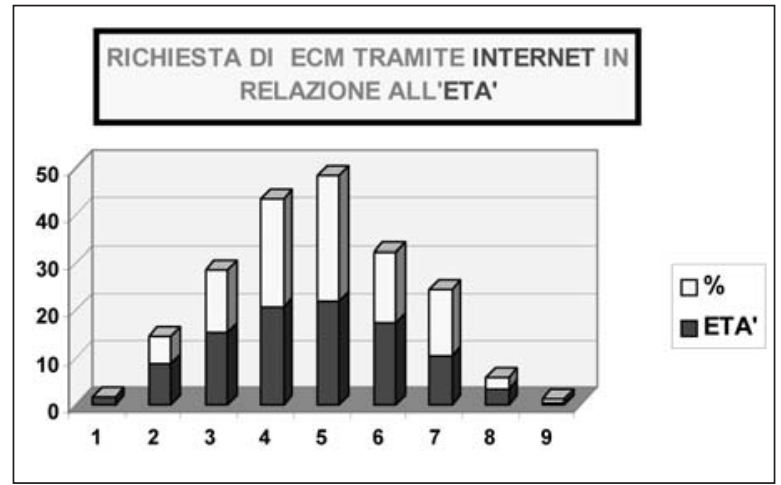

Grafico 12.

In ascissa i numeri $1,2,3 \ldots \ldots$ indicano le classi di età. La parte inferiore dell'istogramma (colonne blu) indica la distribuzione dell'età dei tecnici che hanno risposto (vedi Grafico 2), mentre la parte superirore (colonne gialle) la percentuale di coloro che hanno espresso la preferenza esclusiva o combinata (vedi questionario) per l'utilizzo della formazione a distanza. Il dato da sottolineare è la distribuzione omogenea per tutte le classi di età il che sottolinea che l'utilizzo di mezzi, quali internet, come ci si potrebbe aspettare, non è solo appannaggio dei più giovani. I nuovi mezzi di comunicazione costituiscono quindi uno stimolo per tutti. É pertanto prevedibile che in un prossimo futuro questo tipo di aggiornamento acquisirà sempre più importanza e consensi.

A chiusura del questionario vi sono state alcune note che richiamano l'attenzione sul fatto che l'ECM deve essere qualitativamente ottimale, non ripetitiva e finalizzata al miglioramento della qualità del lavoro, deve avere costi contenuti e gli eventi dovrebbero essere organizzati in sedi vicine al posto di lavoro ed in orario di servizio.

\section{CONCLUSIONI}

L'analisi dei questionari ha evidenziato un panorama molto variegato sia per quanto riguarda la scolarità del personale tecnico, sia per quanto riguarda la tipologia dei laboratori di appartenenza.

Emerge la necessità di approfondimento delle conoscenze nel settore in cui viene svolto il lavo- 
ro quotidiano ed un elevato gradimento trasversale per la biologia molecolare. Nell'ambito degli argomenti specifici da trattare le segnalazioni maggiori sono per il controllo di qualità, il rischio biologico e le infezioni ospedaliere. Le procedure di accreditamento e/o di certificazione che già in molti laboratori sono state attuate, oltre alle problematiche che si sono presentate con le nuove tecnologie (standardizzazione ecc.) sono indubbiamente i fattori che hanno contribuito maggiormente a far convogliare l'attenzione sul problema del controllo di qualità.

La sensibilizzazione nei confronti del rischio biologico è invece legata all'attuazione del D.L. 626/94 e successive modifiche. Questo decreto ha introdotto un concetto nuovo per quanto riguarda la tutela della salute degli operatori: il soggetto non è più parte passiva ma attiva e, una volta adeguatamente formato e dotato dei dispositivi di protezione adatti deve lui stesso provvedere alla sua salute seguendo le indicazioni che gli sono state date e segnalando al responsabile della struttura qualsiasi problema possa presentarsi. Nel caso vi sia un incidente e chi lo ha subito non ha seguito le norme di sicurezza che gli sono state insegnate, responsabile dell'accaduto è lui stesso. Essendoci pertanto un coinvolgimento attivo degli operatori vi è presumibilmente una maggiore attenzione verso la tutela della propria salute. Le infezioni ospedaliere sono argomento di grande attualità: la costituzione del CIO (Comitato Infezioni Ospedaliere) e l'abbondanza di lavori scientifici che si possono reperire sull'argomento ne sono una chiara testimonianza. A ciò si associa il contributo sempre maggiore che la Microbiologia può dare in questo campo grazie anche alle possibilità offerte dalle nuove tecnologie (es. epidemiologia molecolare).

La formazione teorico-pratica è quella più richiesta e sottolinea la necessità del tecnico di conoscere e capire quello che fa routinariamente e non solo di essere un esecutore.

Nell'ambito degli argomenti generali le innovazioni tecnologiche sono quello sul quale si sono maggiormente concentrate le preferenze. Esse infatti non solo aumentano le possibilità diagnostiche ma sono anche fondamentali per il miglioramento del lavoro sia dal punto di vista operativo che qualitativo. Consentono di razionalizzarlo e di affrontarlo in modo sempre più agevole.

Sorprendentemente non è emerso un gradimento elevato per la formazione "a distanza": questo è probabilmente dovuto al fatto che ancora non è ancora stata avviata né sono chiare le modalità con cui verrà svolta. L'interesse per il suo utilizzo, distribuito uniformemente nell'ambito di tutte le classi di età porta a pensare che nel momento in cui verrà codificata probabilmente troverà maggiori consensi.

In conclusione l'indagine ci ha consentito di avere un quadro verosimilmente attendibile dei fabbisogni formativi dei tecnici e ci permetterà di costruire gradualmente un progetto formativo di qualità, efficace $\mathrm{e}$ che non potrà che avere ricadute positive sulla qualità totale dei risultati che giornalmente vengono forniti al clinico.

\section{BIBLIOGRAFIA}

1. Brigley S, Young Y, Littlejohns P, McEwen J. Continuing education for medical professionals: a reflective model. Postgr Med 1997; 73 (855): 23-9.

2. Colliera CP, Crowe AT, Stintson RA, Chu SY, Houlden RL. The continuing professional development of the Canadian Society of Clinical Chemists and the Canadian Academy of Clinical Biochemists. Clin Biochem 2001; 34 (2): 91-6.

3. Diamandis EP. Duties and responsabilities of laboratory scientist. Clin Chem Acta 2002; 319 (2): 111-5.

4. Dominiczack MH. Teaching and training laboratory professionals for the 21 st century. Clin Chem Lab Med 1988; 36 (3): 133-6.

5. Dunne MW, Pinckard JK, Hooper L. Clinical microbiology in the year 2025. Jour of Clin Microbiol 2002; 40 (11): 3889-93.

6. Edmonstone J, Havergal M. The third way: a new approach to management education in health care. Health Manpow Manage 1998; 24 (1): 33-7.

7. Hicks B. Lessons from the Bristol Inquiry for education and training in the NHS. Hosp Med 2001: 62 (9): 556-9.

8. Iwatani Y. Clinical laboratory physicians and postgraduate training for laboratory. Rinsho Byori 2000; 48 (9): 858-9.

9. Kumasaka K. External quality assessment for clinical microbiology and good laboratory management. Rinsho Byori 1998; 46 (2): 124-31.

10. Kumasaka K. What should a laboratory physician expect from a microbiology laboratory?. Rinsho Byori 2000; 48 (10): 926-30.

11. Morozova VT, Dolgov VV, Malakhov VN. Training of clinical laboratory professionals in Russia. Clin Chem Acta 1994; 232 (1-2): 167-71.

12. Ohsawa S. The perspective of medical technologist. Rinsho Byori 1998; 46 (10): 999-1002.

13. Pansini N. The national health system: future possibilities for the clinical laboratory. Clin Chim Acta 2002; 319 (2): 101-5

14. Pfaller A. Molecular approaches to diagnosing and managing infectious diseases: practically and costs. Emerg Infect Dis 2001; 7 (2): 312-8.

15. Plebani $M$. The changing face of clinical laboratories. Clin Chem Lab Med 1999; 37 (7): 711-7.

16. Plebani M. Continuing medical education: a challenge to Italian Scientific Societies of Laboratory Medicine. Clin Chim Acta 2002; 319 (2): 161-7.

17. Pressato L. The italian way to Continuing Education in Medicine.Clin Chim Acta 2002; 319 (2): 155-9.

18. Rosner ER, Reiss E, Warren NG, Shadomy HJ, Lipman HB. Evaluation of the status of laboratory prectices and the need for continuing education in medical mycology. Am J Clin Pathol 2002; 118 (2): 278-86. 
19. Suter E, Green JS, Lawrence K, Walthall DB. Continuing education of health professionals: proposal for a definition of quality. J Med Educ 1981; 56 (8): 687-707.

20. Wood J. The role, duties and responsabilities of technologists inthe clinical laboratory. Clin Chem Acta 2002; 319 (2): 127-32.

Marina Crovatto

Struttura Operativa Semplice di Immunologia Clinica e Virologia

Dipartimento di Medicina di Laboratorio Azienda Ospedaliera Santa Maria degli Angeli

Via Montereale 24, 33170 Pordenone

Tel. 0434399608 - Cell. 339-8666974

Fax 0434399685

E-mail: marina.crovatto@libero.it 